\title{
GROWTH ENVELOPES OF ANISOTROPIC FUNCTION SPACES
}

\author{
SUSANA D. MOURA, JÚLIO S. NEVES AND MARIUSZ PIOTROWSKI \\ Dedicated to Professor H. Triebel on the occasion of his 70th birthday
}

\begin{abstract}
The present paper is devoted to the study of growth envelopes of anisotropic function spaces. An affirmative answer is given to the question of [19, Conjecture 13], whether the growth envelopes are independent of anisotropy. As an application, related anisotropic Hardy inequalities are presented and we also discuss a connection to some anisotropic fractal sets.
\end{abstract}

KEYWORDS: anisotropic function spaces, growth envelopes, Hardy inequalities. AMS Subject Classification (2000): 46E35, 42E35.

\section{Introduction}

The concept of envelopes in function spaces turns out to be an important tool for studying spaces of Besov or Triebel-Lizorkin type in limiting situations. The investigation of envelopes of function spaces was initiated by D.D. Haroske in [10] and H. Triebel in [17]. A systematic study of this concept started only rather recently, see, e.g. [17, Chapter II], [11] and [12]. The interested reader is referred to the monograph [17] for further information on the history of this concept. For recent contributions on growth envelopes of spaces of generalized smoothness we refer to [6], [7], [3], [4] and [5].

In this work we consider growth envelopes of anisotropic Besov and TriebelLizorkin spaces in the subcritical and critical case. The main purpose of this paper is to give an affirmative answer to the conjecture posed by $\mathrm{H}$. Triebel in [19], where the question was raised whether the results on growth envelopes for anisotropic function spaces are affected by anisotropy.

Let us now present the contents of this work in some detail. The next section collects the necessary background material. In Subsection 2.1 some general notation is introduced. Subsection 2.2 covers results from the theory of anisotropic function spaces, namely interpolation properties, embeddings

Received 19th March 2006.

This research was supported by CMUC. 
and characterization by atomic decompositions whereas Subsection 2.3 collects the fundamentals on growth envelopes. On Section 3 we present some embedding results which are useful when discussing growth envelopes for anisotropic function spaces. Section 4 is devoted to the study of growth envelopes of anisotropic function spaces in the subcritical case and Section 5 deals with the critical case. In both cases we prove that the growth envelopes are independent of the anisotropy. The last section deals with related anisotropic Hardy type inequalities and a connection to some anisotropic fractal sets.

\section{Preliminaries}

2.1. General Notation. For a real number $a,[a]$ stands for the greatest integer less than or equal to $a$ and let $a_{+}:=\max (a, 0)$. By $c, c_{1}, c_{2}$, etc. we denote positive constants independent of appropriate quantities. For two non-negative expressions (i.e. functions or functionals) $\mathcal{A}, \mathcal{B}$, the symbol $\mathcal{A} \lesssim \mathcal{B}$ (or $\mathcal{A} \gtrsim \mathcal{B}$ ) means that $\mathcal{A} \leq c \mathcal{B}$ (or $c \mathcal{A} \geq \mathcal{B}$ ). If $\mathcal{A} \lesssim \mathcal{B}$ and $\mathcal{A} \gtrsim \mathcal{B}$, we write $\mathcal{A} \sim \mathcal{B}$ and say that $\mathcal{A}$ and $\mathcal{B}$ are equivalent. For $p \in[1, \infty]$, the conjugate number $p^{\prime}$ is defined by $1 / p+1 / p^{\prime}=1$ with the convention that $1 / \infty=0$. Given two quasi-Banach spaces $X$ and $Y$, we write $X \hookrightarrow Y$ if $X \subset Y$ and the natural embedding is bounded. In the following let both $\mathrm{d} x$ and $|\cdot|$ stand for the Lebesgue measure in $\mathbb{R}^{n}$. Recall that the distribution function $\mu_{f}(\lambda)$ and the non-increasing rearrangement $f^{*}$ of a complex-valued Lebesgue measurable function $f$ are given by

$$
\mu_{f}(\lambda):=\left|\left\{x \in \mathbb{R}^{n}:|f(x)|>\lambda\right\}\right|
$$

and

$$
f^{*}(t):=\inf \left\{\lambda: \mu_{f}(\lambda) \leq t\right\}, \quad t \geq 0,
$$

respectively. Analogously, for a complex-valued sequence $\left(a_{m}\right)_{m \in \mathbb{Z}^{n}}$, its nonincreasing rearrangement is given as the sequence $\left(a_{l}^{*}\right)_{l \in \mathbb{N}}$, where

$$
a_{l}^{*}:=\inf \left\{\lambda \geq 0: \#\left\{m \in \mathbb{Z}^{n}:\left|a_{m}\right|>\lambda\right\}<l\right\}, \quad l \in \mathbb{N} .
$$

Let $0<r, v \leq \infty$. Then the Lorentz space $L_{r v}\left(\mathbb{R}^{n}\right)$ consists of all Lebesgue measurable complex-valued functions $f$ on $\mathbb{R}^{n}$ for which the quasi-norm 


$$
\left\|f \mid L_{r v}\left(\mathbb{R}^{n}\right)\right\|:=\left\{\begin{array}{lll}
\left(\int_{0}^{\infty}\left(t^{1 / r} f^{*}(t)\right)^{v} \frac{\mathrm{d} t}{t}\right)^{1 / v}, & \text { for } & 0<v<\infty \\
\sup _{t>0} t^{1 / r} f^{*}(t), & \text { for } & v=\infty
\end{array}\right.
$$

is finite.

2.2. Anisotropic function spaces. In this subsection we introduce the anisotropic Besov and Triebel-Lizorkin spaces and describe some important properties. Let us start by recalling briefly the basic ingredients needed to introduce these spaces by the Fourier-analytical approach. Throughout the paper we call the vector

$$
\alpha=\left(\alpha_{1}, \ldots, \alpha_{n}\right) \quad \text { with } \quad 0<\alpha_{1} \leq \ldots \leq \alpha_{n}<\infty \quad \text { and } \quad \sum_{j=1}^{n} \alpha_{j}=n
$$

an anisotropy in $\mathbb{R}^{n}$. For $t>0, r \in \mathbb{R}$ and $x=\left(x_{1}, \ldots, x_{n}\right) \in \mathbb{R}^{n}$ we put

$$
t^{\alpha} x:=\left(t^{\alpha_{1}} x_{1}, \ldots t^{\alpha_{n}} x_{n}\right) \text { and } t^{r \alpha} x:=\left(t^{r}\right)^{\alpha} x .
$$

For $x=\left(x_{1}, \ldots, x_{n}\right) \in \mathbb{R}^{n}, x \neq 0$, let $|x|_{\alpha}$ be the unique positive number $t$ such that

$$
\frac{x_{1}^{2}}{t^{2 \alpha_{1}}}+\ldots+\frac{x_{n}^{2}}{t^{2 \alpha_{n}}}=1
$$

and put $|0|_{\alpha}=0$. It turns out that $|\cdot|_{\alpha}$ is an anisotropic distance function in $C^{\infty}\left(\mathbb{R}^{n}\right) \backslash\{0\}$ according to [8, Definition 2.1]. Note that in the isotropic case, which means $\alpha_{1}=\cdots=\alpha_{n}=1,|x|_{\alpha}$ is the Euclidean distance of $x$ to the origin.

Let $\varphi_{0}^{\alpha} \in \mathcal{S}\left(\mathbb{R}^{n}\right)$ be a function such that

$$
\varphi_{0}^{\alpha}(x)=1 \quad \text { for } \quad \sup _{l}\left|x_{l}\right| \leq 1 \quad \text { and } \quad \varphi_{0}^{\alpha}(y)=0 \quad \text { for } \quad \sup _{l} 2^{-\alpha_{l}}\left|y_{l}\right| \geq 1
$$

with $x=\left(x_{1}, \ldots, x_{n}\right) \in \mathbb{R}^{n}$ and $y=\left(y_{1}, \ldots, y_{n}\right) \in \mathbb{R}^{n}$. We define

$$
\varphi_{j}^{\alpha}(x):=\varphi_{0}^{\alpha}\left(2^{-j \alpha} x\right)-\varphi_{0}^{\alpha}\left(2^{-(j-1) \alpha} x\right), \quad x \in \mathbb{R}^{n}, \quad j \in \mathbb{N} .
$$


Then since $\sum_{j=0}^{\infty} \varphi_{j}^{\alpha}(x)=1$ for all $x \in \mathbb{R}^{n}$, the sequence $\left(\varphi_{j}^{\alpha}\right)_{j \in \mathbb{N}_{0}}$ is an anisotropic resolution of unity with

$$
\operatorname{supp} \varphi_{0}^{\alpha} \subset R_{1}^{\alpha} \text { and } \operatorname{supp} \varphi_{j}^{\alpha} \subset R_{j+1}^{\alpha} \backslash R_{j-1}^{\alpha}, \quad j \in \mathbb{N},
$$

where $R_{j}^{\alpha}$ are rectangles given by

$$
R_{j}^{\alpha}:=\left\{x:\left|x_{l}\right| \leq 2^{\alpha_{l} j}, l=1, \ldots, n\right\}, \quad j \in \mathbb{N}_{0} .
$$

Definition 2.1. Let $\alpha$ be an anisotropy as in (2) and let $\varphi^{\alpha}=\left(\varphi_{j}^{\alpha}\right)_{j \in \mathbb{N}_{0}}$ be an anisotropic dyadic resolution of unity in the sense of (4).

(i) For $0<p, q \leq \infty$ and $s \in \mathbb{R}$ the anisotropic Besov space $B_{p q}^{s, \alpha}\left(\mathbb{R}^{n}\right)$ is defined to be the set of all tempered distributions $f \in \mathcal{S}^{\prime}\left(\mathbb{R}^{n}\right)$ such that

$$
\left\|f \mid B_{p q}^{s, \alpha}\left(\mathbb{R}^{n}\right)\right\|:=\left(\sum_{j=0}^{\infty} 2^{j s q}\left\|\left(\varphi_{j}^{\alpha} \widehat{f}\right)^{\vee} \mid L_{p}\left(\mathbb{R}^{n}\right)\right\|^{q}\right)^{1 / q}
$$

is finite. In the limiting case $q=\infty$ the usual modification is required.

(ii) For $0<p<\infty, 0<q \leq \infty$ and $s \in \mathbb{R}$ the anisotropic Triebel-Lizorkin space $F_{p q}^{s, \alpha}\left(\mathbb{R}^{n}\right)$ is defined to be the set of all tempered distributions $f \in$ $\mathcal{S}^{\prime}\left(\mathbb{R}^{n}\right)$ such that

$$
\left\|f\left|F_{p q}^{s, \alpha}\left(\mathbb{R}^{n}\right)\|:=\|\left(\sum_{j=0}^{\infty} 2^{j s q}\left|\left(\varphi_{j}^{\alpha} \widehat{f}\right)^{\vee}(\cdot)\right|^{q}\right)^{1 / q}\right| L_{p}\left(\mathbb{R}^{n}\right)\right\|
$$

is finite. In the limiting case $q=\infty$ the usual modification is required.

Remark 2.2. We occasionally use the symbol $A_{p q}^{s, \alpha}\left(\mathbb{R}^{n}\right)$ to consider the spaces $B_{p q}^{s, \alpha}\left(\mathbb{R}^{n}\right)$ and $F_{p q}^{s, \alpha}\left(\mathbb{R}^{n}\right)$ simultaneously. It turns out that $A_{p q}^{s, \alpha}\left(\mathbb{R}^{n}\right)$ are quasiBanach spaces which are independent of $\varphi^{\alpha}$, in the sense of equivalent quasinorms, according to either (5) or (6). Taking $\alpha=(1, \ldots, 1)$ brings us back to the isotropic case usually denoted by $B_{p q}^{s}\left(\mathbb{R}^{n}\right)$ and $F_{p q}^{s}\left(\mathbb{R}^{n}\right)$. The above Fourier analytical approach is due to H. Triebel [16]. For further information the reader may consult [13] and [18], specially in the later monograph one can find historical considerations.

We start by stating an anisotropic Littlewood-Paley theorem. Details may be found in $[13,4.2 .2]$.

Proposition 2.3. Let $\alpha$ be an anisotropy as in (2) and let $1<p<\infty$. Then

$$
F_{p, 2}^{0, \alpha}\left(\mathbb{R}^{n}\right)=L_{p}\left(\mathbb{R}^{n}\right) .
$$


Let us continue by describing the interpolation property of anisotropic Besov spaces according to [9, Appendix C.2, Lemma 2].

Proposition 2.4. Let $\alpha$ be an anisotropy as in (2). Furthermore, let $0<$ $q_{0}, q_{1} \leq \infty, s_{0}, s_{1} \in \mathbb{R}$ such that $s_{0} \neq s_{1}$ and let $s=(1-\theta) s_{0}+\theta s_{1}$ with $0<\theta<1$. Then

$$
\left(B_{p q_{0}}^{s_{0}, \alpha}\left(\mathbb{R}^{n}\right), B_{p q_{1}}^{s_{1}, \alpha}\left(\mathbb{R}^{n}\right)\right)_{\theta, q}=B_{p q}^{s, \alpha}\left(\mathbb{R}^{n}\right) \quad \text { for all } \quad 0<p, q \leq \infty .
$$

We refer to [9, Appendix C.3, Proposition 7] for the next assertion on embeddings between anisotropic Besov and Triebel-Lizorkin spaces.

Proposition 2.5. Let $\alpha$ be an anisotropy as in (2). Let $0<p_{0}<p<p_{1} \leq$ $\infty, s_{1}<s<s_{0}$ and $0<q \leq \infty$. Then

$$
B_{p_{0} p}^{s_{0}, \alpha}\left(\mathbb{R}^{n}\right) \hookrightarrow F_{p q}^{s, \alpha}\left(\mathbb{R}^{n}\right) \hookrightarrow B_{p_{1} p}^{s_{1}, \alpha}\left(\mathbb{R}^{n}\right),
$$

provided that $s_{0}-n / p_{0}=s-n / p=s_{1}-n / p_{1}$.

Let us proceed with a review of the needed notation to state the atomic decomposition result. Hereby, we closely follow the exposition of W. Farkas from [8]. Let $\alpha$ be an anisotropy as in (2). Let $Q_{\nu m}^{\alpha}$ be the rectangle in $\mathbb{R}^{n}$ with sides parallel to the axes of coordinates, centered at

$$
2^{-\nu \alpha} m=\left(2^{-\nu \alpha_{1}} m_{1}, \ldots, 2^{-\nu \alpha_{n}} m_{n}\right), \quad m \in \mathbb{Z}^{n}, \nu \in \mathbb{N}_{0},
$$

and with sides lengths $2^{-(\nu-1) \alpha_{1}}, \ldots, 2^{-(\nu-1) \alpha_{n}}$. In particular, $Q_{0 m}^{\alpha}$ are rectangles of sides lengths $2^{\alpha_{1}}, \ldots, 2^{\alpha_{n}}$ centered at $m \in \mathbb{Z}^{n}$. If $Q_{\nu m}^{\alpha}$ is such a rectangle in $\mathbb{R}^{n}$ and $d>0$ then $d Q_{\nu m}^{\alpha}$ is the rectangle in $\mathbb{R}^{n}$ concentric with $Q_{\nu m}^{\alpha}$ and with side lengths $d 2^{-(\nu-1) \alpha_{1}}, \ldots, d 2^{-(\nu-1) \alpha_{n}}$.

Remark that for fixed $\nu \in \mathbb{N}_{0}$ and $d>0$, there are at most $(2 d)^{n}$ cubes $d Q_{\nu m}^{\alpha}, m \in \mathbb{N}$, that overlap. For $x \in \mathbb{R}^{n}$ and $\gamma \in \mathbb{N}_{0}^{n}$ we put

$$
\alpha \gamma=\gamma \alpha:=\sum_{j=1}^{n} \gamma_{j} \alpha_{j} \quad \text { and } \quad x^{\gamma}:=x_{1}^{\gamma_{1}} \ldots x_{n}^{\gamma_{n}} .
$$

We are now in a position to introduce the respective building blocks.

Definition 2.6. Let $\alpha$ be an anisotropy according to (2). Let $K \geq 0$ and $d>1$. A continuous function $a: \mathbb{R}^{n} \rightarrow \mathbb{C}$ with all derivatives $\mathrm{D}^{\gamma} a$ for $\alpha \gamma \leq K$ is said to be an $1_{K}^{\alpha}$-atom if

(i) supp $a \subset d Q_{0 m}^{\alpha} \quad$ for some $\quad m \in \mathbb{Z}^{n}$, 
(ii) $\left|\mathrm{D}^{\gamma} a(x)\right| \leq 1 \quad$ for $\quad \alpha \gamma \leq K, \quad x \in \mathbb{R}^{n}$.

Let $s \in \mathbb{R}, 0<p \leq \infty, K, L \geq 0$ and $d>1$. A continuous function $a: \mathbb{R}^{n} \rightarrow \mathbb{C}$ with all derivatives $\mathrm{D}^{\gamma} a$ for $\alpha \gamma \leq K$ is said to be an $(s, p)_{K, L^{-}}^{\alpha}$ atom if

(i) $\operatorname{supp} a \subset d Q_{\nu m}^{\alpha} \quad$ for some $\quad \nu \in \mathbb{N}_{0}, m \in \mathbb{Z}^{n}$,

(ii) $\left|\mathrm{D}^{\gamma} a(x)\right| \leq 2^{-\nu\left(s-\frac{n}{p}-\gamma \alpha\right)}$ for $\alpha \gamma \leq K, \quad x \in \mathbb{R}^{n}$,

(iii) $\int_{\mathbb{R}^{n}} x^{\beta} a(x) \mathrm{d} x=0 \quad$ for $\quad \beta \in \mathbb{N}_{0}^{n} \quad$ with $\beta \alpha<L$.

Definition 2.7. If $0<p, q \leq \infty$, the Besov sequence space $b_{p q}$ is the collection of all sequences $\lambda=\left(\lambda_{\nu m}\right)_{\nu \in \mathbb{N}_{0}, m \in \mathbb{Z}^{n}} \subset \mathbb{C}$ such that

$$
\left\|\lambda \mid b_{p q}\right\|:=\left(\sum_{\nu=0}^{\infty}\left(\sum_{m \in \mathbb{Z}^{n}}\left|\lambda_{\nu m}\right|^{p}\right)^{q / p}\right)^{1 / q}
$$

(with the usual modification if either $p=\infty$ or $q=\infty$ ) is finite.

In the sequel, to shorten the notation, we use the following abbreviation

$$
\sigma_{p}:=n\left(\frac{1}{p}-1\right)_{+} .
$$

Below we formulate the atomic decomposition of anisotropic Besov spaces $B_{p q}^{s, \alpha}\left(\mathbb{R}^{n}\right)$ as presented in [8, Theorem 3.3].

Theorem 2.8. Let $0<p, q \leq \infty, s \in \mathbb{R}$ and $\alpha$ be an anisotropy according to (2). Let $K, L \geq 0$ with

$$
K \geq\left\{\begin{array}{lll}
0 & \text { for } & s<0 \\
s+\alpha_{n} & \text { for } & s \geq 0
\end{array}\right.
$$

and $L>\sigma_{p}-s$ be fixed.

A tempered distribution $f \in \mathcal{S}^{\prime}\left(\mathbb{R}^{n}\right)$ belongs to $B_{p q}^{s, \alpha}\left(\mathbb{R}^{n}\right)$ if, and only if, it can be written as

$$
f=\sum_{\nu=0}^{\infty} \sum_{m \in \mathbb{Z}^{n}} \lambda_{\nu m} a_{\nu m}^{\alpha}, \quad \text { converging in } \mathcal{S}^{\prime}\left(\mathbb{R}^{n}\right),
$$

where $a_{\nu m}^{\alpha}$ are $1_{K}^{\alpha}$-atoms $(\nu=0)$ or $(s, p)_{K, L^{-a t o m s}}^{\alpha}(\nu \in \mathbb{N})$ and $\lambda \in b_{p q}$. Furthermore

$$
\inf \left\|\lambda \mid b_{p q}\right\|
$$


where the infimum is taken over all admissible representations (10), is an equivalent quasi-norm in $B_{p q}^{s, \alpha}\left(\mathbb{R}^{n}\right)$.

2.3. Growth envelopes. The notion of growth envelopes was introduced by D. D. Haroske in [10] and H. Triebel in [17]. Following [10], we present the basic definitions and properties concerning growth envelopes in quasi-Banach function spaces in the sense of [1]. However, we shall be rather concise and we mainly refer to $[10,11,17,12]$ for heuristics, motivations and details on this subject.

Definition 2.9. Let $X$ be some quasi-Banach function space on $\mathbb{R}^{n}$.

(i) The growth envelope function $\mathcal{E}_{\mathrm{G}} X:(0, \infty) \rightarrow[0, \infty)$ is defined by

$$
\mathcal{E}_{\mathrm{G}} X(t):=\sup _{\|f \mid X\| \leq 1} f^{*}(t), \quad t>0
$$

(ii) Assume $X \nrightarrow L_{\infty}\left(\mathbb{R}^{n}\right)$. Let $\varepsilon \in(0,1), H(t):=-\log \mathcal{E}_{\mathrm{G}} X(t), t \in(0, \varepsilon]$, and let $\mu_{H}$ be the associated Borel measure. The number $u_{X}, 0<$ $u_{X} \leq \infty$, is defined as the minimum (assuming it exists) of all numbers $v, 0<v \leq \infty$, such that

$$
\left(\int_{0}^{\varepsilon}\left(\frac{f^{*}(t)}{\mathcal{E}_{\mathrm{G}} X(t)}\right)^{v} \mu_{H}(\mathrm{~d} t)\right)^{1 / v} \leq c\|f \mid X\|
$$

(with the usual modification if $v=\infty$ ) holds for some $c>0$ and all $f \in X$. The couple

$$
\mathfrak{E}_{\mathrm{G}}(X)=\left(\mathcal{E}_{\mathrm{G}} X(\cdot), u_{X}\right)
$$

is called growth envelope for the function space $X$.

As it will be useful in the sequel, we recall some properties of the growth envelopes. In view of Definition 2.9 (i) we obtain - strictly speaking - equivalence classes of growth envelope functions when working with equivalent (quasi-) norms in $X$. However, for convenience we do not want to distinguish between representative and equivalence class in what follows and thus stick at the notation introduced in Definition $2.9(\mathrm{i})$. Note that $\mathcal{E}_{\mathrm{G}} X$ is a 
monotonically decreasing function. Concerning Definition 2.9 (ii) it is obvious that (13) holds for $v=\infty$ and any $X$. Moreover, one verifies that

$$
\begin{aligned}
\sup _{0<t \leq \varepsilon} \frac{g(t)}{\mathcal{E}_{\mathrm{G}} X(t)} & \leq c_{1}\left(\int_{0}^{\varepsilon}\left(\frac{g(t)}{\mathcal{E}_{\mathrm{G}} X(t)}\right)^{v_{1}} \mu_{H}(\mathrm{~d} t)\right)^{1 / v_{1}} \\
& \leq c_{2}\left(\int_{0}^{\varepsilon}\left(\frac{g(t)}{\mathcal{E}_{\mathrm{G}} X(t)}\right)^{v_{0}} \mu_{H}(\mathrm{~d} t)\right)^{1 / v_{0}}
\end{aligned}
$$

for $0<v_{0}<v_{1}<\infty$ and all non-negative monotonically decreasing functions $g$ on $(0, \varepsilon]$; cf. [17, Proposition 12.2, p. 183-184]. So we observe that the lefthand sides in (13) are monotonically ordered in $v$ and it is natural to look for the smallest possible one.

Proposition 2.10. (i) Let $X$ be some quasi-Banach function space on $\mathbb{R}^{n}$. Then $X \hookrightarrow L_{\infty}\left(\mathbb{R}^{n}\right)$ if, and only if, $\mathcal{E}_{\mathrm{G}} X(\cdot)$ is bounded.

(ii) Let $X_{i}, i=1,2$, be some quasi-Banach function spaces on $\mathbb{R}^{n}$. Then $X_{1} \hookrightarrow X_{2}$ implies that there is some positive constant $c$ such that for all $t>0$

$$
\mathcal{E}_{\mathrm{G}} X_{1}(t) \leq c \mathcal{E}_{\mathrm{G}} X_{2}(t)
$$

(iii) Let $X_{i}, i=1,2$, be some quasi-Banach function spaces on $\mathbb{R}^{n}$ with $X_{1} \hookrightarrow X_{2}$. Assume that

$$
\mathcal{E}_{\mathrm{G}} X_{1}(t) \sim \mathcal{E}_{\mathrm{G}} X_{2}(t), \quad t \in(0, \varepsilon),
$$

for some $\varepsilon>0$. Then, the corresponding indices $u_{X_{i}}, i=1,2$, satisfy

$$
u_{X_{1}} \leq u_{X_{2}}
$$

For the proof of the previous proposition and further properties of growth envelopes we refer to [10], in particular to Propositions 2.4 and 3.5, and the forthcoming book [12].

\section{Embeddings into $L_{1}^{\text {loc }}\left(\mathbb{R}^{n}\right)$ and $L_{\infty}\left(\mathbb{R}^{n}\right)$}

In this section we study some embedding results which will be useful when discussing growth envelopes for anisotropic function spaces.

The proposition below gives a sufficient condition under which the anisotropic spaces of Besov and Triebel-Lizorkin type consist only of regular distributions. For a complete characterization of the inclusion of the isotropic 
spaces of Besov and Triebel-Lizorkin type into $L_{1}^{\text {loc }}\left(\mathbb{R}^{n}\right)$ we refer to [14, Theorem 3.3.2].

Proposition 3.1. Let $0<p, q \leq \infty$ (with $p<\infty$ in the $F$-case), $s \in \mathbb{R}$ and $\alpha$ be an anisotropy according to (2). If $s>\sigma_{p}$ then it holds

$$
A_{p q}^{s, \alpha}\left(\mathbb{R}^{n}\right) \subset L_{1}^{\mathrm{loc}}\left(\mathbb{R}^{n}\right) .
$$

Proof: Using the same strategy as in the proof of [14, Theorem 3.3.2], we shall have established the proposition if we prove that

$$
A_{p q}^{s, \alpha}\left(\mathbb{R}^{n}\right) \hookrightarrow L_{\max (1, p)}\left(\mathbb{R}^{n}\right) \text { for } s>\sigma_{p} .
$$

We shall prove this first for $A=B$. Let $s>\sigma_{p}$ and let $f \in B_{p q}^{s, \alpha}\left(\mathbb{R}^{n}\right)$. By Theorem 2.8,

$$
f=\sum_{\nu=0}^{\infty} \sum_{m \in \mathbb{Z}^{n}} \lambda_{\nu m} a_{\nu m}^{\alpha},
$$

where $\lambda=\left(\lambda_{\nu m}\right) \in b_{p q}$ and $a_{\nu m}^{\alpha}$ are $1_{K}$-atoms $(\nu=0)$ or $(s, p)_{K, L}^{\alpha}$ - atoms $(\nu \in \mathbb{N})$, according to Definition 2.6.

Let first $1<p<\infty$. Then $s>0$ and choose $\varepsilon \in(0, s)$. For $L, M \in \mathbb{N}$ with $M>L$, we have

$$
\begin{aligned}
\| \sum_{\nu=L}^{M} \sum_{m \in \mathbb{Z}^{n}} & \lambda_{\nu m} a_{\nu m}^{\alpha}(\cdot) \mid L_{p}\left(\mathbb{R}^{n}\right) \|^{p} \leq \int_{\mathbb{R}^{n}}\left(\sum_{\nu=L}^{M} \sum_{m \in \mathbb{Z}^{n}}\left|\lambda_{\nu m}\right| 2^{-\nu(s-n / p)} \chi_{d Q_{\nu m}^{\alpha}}(x)\right)^{p} \mathrm{~d} x \\
& \leq \int_{\mathbb{R}^{n}}\left(\sum_{\nu=L}^{M} 2^{-\varepsilon \nu p^{\prime}}\right)^{p / p^{\prime}} \sum_{\nu=L}^{M} 2^{-\nu(s-n / p-\varepsilon) p}\left(\sum_{m \in \mathbb{Z}^{n}}\left|\lambda_{\nu m}\right| \chi_{d Q_{\nu m}^{\alpha}}(x)\right)^{p} \mathrm{~d} x \\
& \leq c_{1}\left(\sum_{\nu=L}^{M} 2^{-\varepsilon \nu p^{\prime}}\right)^{p / p^{\prime}} \sum_{\nu=L}^{M} 2^{-\nu(s-n / p-\varepsilon) p} \int_{\mathbb{R}^{n}} \sum_{m \in \mathbb{Z}^{n}}\left|\lambda_{\nu m}\right|^{p} \chi_{d Q_{\nu m}^{\alpha}}(x) \mathrm{d} x \\
& \leq c_{1}\left(\sum_{\nu=L}^{M} 2^{-\varepsilon \nu p^{\prime}}\right)^{p / p^{\prime}} \sum_{\nu=L}^{M} 2^{-\nu(s-n / p-\varepsilon) p} \sum_{m \in \mathbb{Z}^{n}}\left|\lambda_{\nu m}\right|^{p}\left|d Q_{\nu m}^{\alpha}\right| \\
& \leq c_{2}\left(\sum_{\nu=L}^{M} 2^{-\varepsilon \nu p^{\prime}}\right)^{p / p^{\prime}} \sum_{\nu=L}^{M} 2^{-\nu(s-\varepsilon) p} \sum_{m \in \mathbb{Z}^{n}}\left|\lambda_{\nu m}\right|^{p}
\end{aligned}
$$


where $\chi_{d Q_{\nu m}^{\alpha}}$ denotes the characteristic function of the rectangle $d Q_{\nu m}^{\alpha}$. Note that the constant $c_{1}$ depends only on $p$ and on the number of those rectangles for which each fixed $x \in \mathbb{R}^{n}$ belongs. Thus, since $1 / p<1$, we get

$$
\begin{gathered}
\left\|\sum_{\nu=L}^{M} \sum_{m \in \mathbb{Z}^{n}} \lambda_{\nu m} a_{\nu m}^{\alpha}(\cdot) \mid L_{p}\left(\mathbb{R}^{n}\right)\right\| \leq c\left(\sum_{\nu=L}^{M} 2^{-\varepsilon \nu p^{\prime}}\right)^{1 / p^{\prime}}\left(\sum_{\nu=L}^{M} \sum_{m \in \mathbb{Z}^{n}}\left|\lambda_{\nu m}\right|^{p} 2^{-\nu(s-\varepsilon) p}\right)^{1 / p} \\
\leq c\left(\sum_{\nu=L}^{M} 2^{-\varepsilon \nu p^{\prime}}\right)^{1 / p^{\prime}} \sum_{\nu=L}^{M}\left(\sum_{m \in \mathbb{Z}^{n}}\left|\lambda_{\nu m}\right|^{p}\right)^{1 / p} 2^{-\nu(s-\varepsilon)}
\end{gathered}
$$

Now, if $0<q \leq 1$, the right-hand side of (17) can be estimated from above by

$$
\begin{aligned}
& c\left(\sum_{\nu=L}^{M} 2^{-\varepsilon \nu p^{\prime}}\right)^{1 / p^{\prime}}\left(\sum_{\nu=L}^{M}\left(\sum_{m \in \mathbb{Z}^{n}}\left|\lambda_{\nu m}\right|^{p}\right)^{q / p} 2^{-\nu(s-\varepsilon) q}\right)^{1 / q} \\
& \leq c\left\|\lambda \mid b_{p q}\right\|\left(\sum_{\nu=L}^{M} 2^{-\varepsilon \nu p^{\prime}}\right)^{1 / p^{\prime}}
\end{aligned}
$$

which, since $\varepsilon>0$, enables us to conclude that convergence in (16) is not only in $\mathcal{S}^{\prime}\left(\mathbb{R}^{n}\right)$ but also in $L_{p}\left(\mathbb{R}^{n}\right)$, and hence $f \in L_{p}\left(\mathbb{R}^{n}\right)$.

We now show that the same conclusion can be drawn for $q>1$. By Hölder's inequality, the right-hand side of (17) can be estimated from above by

$$
\begin{aligned}
& c\left(\sum_{\nu=0}^{\infty} 2^{-\varepsilon \nu p^{\prime}}\right)^{1 / p^{\prime}}\left(\sum_{\nu=L}^{M}\left(\sum_{m \in \mathbb{Z}^{n}}\left|\lambda_{\nu m}\right|^{p}\right)^{q / p}\right)^{1 / q}\left(\sum_{\nu=L}^{M} 2^{-\nu(s-\varepsilon) q^{\prime}}\right)^{1 / q^{\prime}} \\
& \leq c\left\|\lambda \mid b_{p q}\right\|\left(\sum_{\nu=L}^{M} 2^{-\nu(s-\varepsilon) q^{\prime}}\right)^{1 / q^{\prime}} .
\end{aligned}
$$

Once more, since $s-\varepsilon>0$, we see that the convergence in (16) is not only in $\mathcal{S}^{\prime}\left(\mathbb{R}^{n}\right)$ but also in $L_{p}\left(\mathbb{R}^{n}\right)$, leading to $f \in L_{p}\left(\mathbb{R}^{n}\right)$.

The same reasoning applies to the case $0<p \leq 1$. For $M, L \in \mathbb{N}$ with $M>L$, we have 


$$
\begin{gathered}
\left\|\sum_{\nu=L}^{M} \sum_{m \in \mathbb{Z}^{n}} \lambda_{\nu m} a_{\nu m}^{\alpha}(\cdot)\left|L_{1}\left(\mathbb{R}^{n}\right) \| \leq \int_{\mathbb{R}^{n}} \sum_{\nu=L}^{M} \sum_{m \in \mathbb{Z}^{n}}\right| \lambda_{\nu m} \mid 2^{-\nu(s-n / p)} \chi_{d Q_{\nu m}^{\alpha}}(x) \mathrm{d} x\right. \\
\leq \sum_{\nu=L}^{M} \sum_{m \in \mathbb{Z}^{n}}\left|\lambda_{\nu m}\right| 2^{-\nu(s-n / p)}\left|d Q_{\nu m}^{\alpha}\right| \leq c \sum_{\nu=L}^{M} \sum_{m \in \mathbb{Z}^{n}}\left|\lambda_{\nu m}\right| 2^{-\nu(s-n / p-n)} \\
\leq c \sum_{\nu=L}^{M} 2^{-\nu(s-n / p-n)}\left(\sum_{m \in \mathbb{Z}^{n}}\left|\lambda_{\nu m}\right|^{p}\right)^{1 / p} .
\end{gathered}
$$

If $0<q \leq 1$, the right-hand side of (18) can be estimated from above by

$c_{1}\left(\sum_{\nu=L}^{M} 2^{-\nu(s-n / p-n) q}\left(\sum_{m \in \mathbb{Z}^{n}}\left|\lambda_{\nu m}\right|^{p}\right)^{q / p}\right)^{1 / q} \leq c_{1}\left(\sum_{\nu=L}^{M}\left(\sum_{m \in \mathbb{Z}^{n}}\left|\lambda_{\nu m}\right|^{p}\right)^{q / p}\right)^{1 / q}$,

which implies, since $\lambda \in b_{p q}$, that the convergence of (16) is not only in $\mathcal{S}^{\prime}\left(\mathbb{R}^{n}\right)$ but also in $L_{1}\left(\mathbb{R}^{n}\right)$.

If $q>1$, using Hölder's inequality, the right-hand side of (18) can be estimated from above by

$$
\begin{gathered}
c_{1}\left(\sum_{\nu=L}^{M}\left(\sum_{m \in \mathbb{Z}^{n}}\left|\lambda_{\nu m}\right|^{p}\right)^{q / p}\right)^{1 / q}\left(\sum_{\nu=L}^{M} 2^{-\nu(s-n / p-n) q^{\prime}}\right)^{1 / q^{\prime}} \\
\leq c_{1}\left\|\lambda \mid b_{p q}\right\|\left(\sum_{\nu=L}^{M} 2^{-\nu(s-n / p-n) q^{\prime}}\right)^{1 / q^{\prime}},
\end{gathered}
$$

and once more, since $s-n / p-n>0$ in this case, the convergence in (16) is not only in $\mathcal{S}^{\prime}\left(\mathbb{R}^{n}\right)$ but also in $L_{1}\left(\mathbb{R}^{n}\right)$, so that $f \in L_{1}\left(\mathbb{R}^{n}\right)$.

If $p=\infty$, then $s>0$ and we get

$$
|f(x)| \leq \sum_{\nu=0}^{\infty} \sum_{m \in \mathbb{Z}^{n}}\left|\lambda_{\nu m}\right| 2^{-\nu s} \chi_{d Q_{\nu m}^{\alpha}}(x) \leq c_{2} \sum_{\nu=0}^{\infty} 2^{-\nu s} \sup _{m \in \mathbb{Z}^{n}}\left|\lambda_{\nu m}\right| .
$$

If $0<q \leq 1$, then the right-hand side of (19) has the upper estimate

$$
c_{2}\left(\sum_{\nu=0}^{\infty} 2^{-\nu s q}\left(\sup _{m \in \mathbb{Z}^{n}}\left|\lambda_{\nu m}\right|\right)^{q}\right)^{1 / q} \leq c_{2}\left\|\lambda \mid b_{\infty q}\right\|
$$


leading to $f \in L_{\infty}\left(\mathbb{R}^{n}\right)$.

If $q>1$, then the right-hand side of (19) can be estimated from above by

$$
c_{2}\left(\sum_{\nu=0}^{\infty} 2^{-\nu s q^{\prime}}\right)^{1 / q^{\prime}}\left(\sum_{\nu=0}^{\infty}\left(\sup _{m \in \mathbb{Z}^{n}}\left|\lambda_{\nu m}\right|\right)^{q}\right)^{1 / q} \leq c_{3}\left\|\lambda \mid b_{\infty q}\right\|
$$

and, once more, $f \in L_{\infty}\left(\mathbb{R}^{n}\right)$.

We shall now be concerned with the proof of (15) for $A=F$. Assuming $s>\sigma_{p}$, there is a $\sigma$ such that $s>\sigma>\sigma_{p}$. Define $p_{1}$ by the equation $s-n / p=\sigma-n / p_{1}$. By virtue of Proposition 2.5 we get

$$
F_{p q}^{s, \alpha}\left(\mathbb{R}^{n}\right) \hookrightarrow B_{p_{1} p}^{\sigma, \alpha}\left(\mathbb{R}^{n}\right) .
$$

We check at once that $\sigma>\sigma_{p_{1}}$. Namely, we have $\sigma=s-n / p+n / p_{1}>$ $-n+n / p_{1}$. Then the desired inclusion follows from what has been proved above.

Regarding the embeddings of the anisotropic spaces into $L_{\infty}\left(\mathbb{R}^{n}\right)$, we have the following:

Proposition 3.2. Let $0<p, q \leq \infty$ and let $\alpha$ be an anisotropy according to (2).

(i) Then

$B_{p q}^{n / p, \alpha}\left(\mathbb{R}^{n}\right) \hookrightarrow L_{\infty}\left(\mathbb{R}^{n}\right) \quad$ if, and only if, $\quad 0<p \leq \infty$ and $0<q \leq 1$.

(ii) Let $0<p<\infty$. Then

$$
F_{p q}^{n / p, \alpha}\left(\mathbb{R}^{n}\right) \hookrightarrow L_{\infty}\left(\mathbb{R}^{n}\right) \quad \text { if, and only if, } \quad 0<p \leq 1 \text { and } 0<q \leq \infty \text {. }
$$

Proof: We only prove here the "if part" as the reverse implications will follow immediately from our results on growth envelopes (see Remark 5.2).

Starting the proof for (i), let $0<q \leq 1$ and let $f \in B_{p q}^{n / p, \alpha}\left(\mathbb{R}^{n}\right)$. By the atomic decomposition theorem (cf. Theorem 2.8)

$$
f=\sum_{\nu=0}^{\infty} \sum_{m \in \mathbb{Z}^{n}} \lambda_{\nu m} a_{\nu m}^{\alpha}
$$

where $\lambda=\left(\lambda_{\nu m}\right) \in b_{p q}$ and $a_{\nu m}^{\alpha}$ are $1_{K}$-atoms $(\nu=0)$ or $(s, p)_{K, L}^{\alpha}$ - atoms $(\nu \in \mathbb{N})$ according to Definition 2.6. Let $x \in \mathbb{R}^{n}$. By the properties of atoms 
we obtain

$$
\begin{aligned}
& |f(x)| \leq \sum_{\nu=0}^{\infty} \sum_{m \in \mathbb{Z}^{n}}\left|\lambda_{\nu m}\right|\left|a_{\nu m}^{\alpha}(x)\right| \leq \sum_{\nu=0}^{\infty} \sum_{\left\{m \in \mathbb{Z}^{n}: x \in d Q_{\nu m}^{\alpha}\right\}}\left|\lambda_{\nu m}\right| \\
& \leq c(p, d, n) \sum_{\nu=0}^{\infty}\left(\sum_{\left\{m \in \mathbb{Z}^{n}: x \in d Q_{\nu m}^{\alpha}\right\}}\left|\lambda_{\nu m}\right|^{p}\right)^{1 / p} \leq c(p, d, n) \sum_{\nu=0}^{\infty}\left(\sum_{m \in \mathbb{Z}^{n}}\left|\lambda_{\nu m}\right|^{p}\right)^{1 / p} \\
& \leq c(p, d, n)\left(\sum_{\nu=0}^{\infty}\left(\sum_{m \in \mathbb{Z}^{n}}\left|\lambda_{\nu m}\right|^{p}\right)^{q / p}\right)^{1 / q} .
\end{aligned}
$$

In the last inequality we have used only the fact that $q \leq 1$. The result of the "if part" of (i) now follows.

To prove the "if part" of (ii), we again apply Proposition 2.5. Assume that $p \leq 1$. By what has been proved above and taking some $p_{1}>p$, we obtain

$$
F_{p q}^{n / p, \alpha}\left(\mathbb{R}^{n}\right) \hookrightarrow B_{p_{1} p}^{n / p_{1}, \alpha}\left(\mathbb{R}^{n}\right) \hookrightarrow L_{\infty}\left(\mathbb{R}^{n}\right)
$$

\section{Growth envelopes of anisotropic function spaces in the subcritical case}

It turns out that the notion of growth envelope, as introduced in Subsection 2.3, makes sense only for anisotropic function spaces of regular distributions. Due to Proposition 3.1 we shall consider only function spaces $A_{p q}^{s, \alpha}\left(\mathbb{R}^{n}\right)$ such that $s>\sigma_{p}$.

This section deals with growth envelopes of anisotropic spaces $A_{p q}^{s, \alpha}\left(\mathbb{R}^{n}\right)$ in the subcritical case whether the critical case is postponed to the next section.

In this section, the spaces of interest for us will be $A_{p q}^{s, \alpha}\left(\mathbb{R}^{n}\right)$ with

$$
\sigma_{p}<s<\frac{n}{p}, \quad 0<p<\infty, \quad \text { and } \quad 0<q \leq \infty
$$

the so-called subcritical case. Let us remark that according to Theorem 4.3 , the growth envelope functions of $A_{p q}^{s, \alpha}\left(\mathbb{R}^{n}\right)$, in the subcritical case, are unbounded and, hence, the embedding $A_{p q}^{s, \alpha}\left(\mathbb{R}^{n}\right) \hookrightarrow L_{\infty}\left(\mathbb{R}^{n}\right)$ does not hold. 


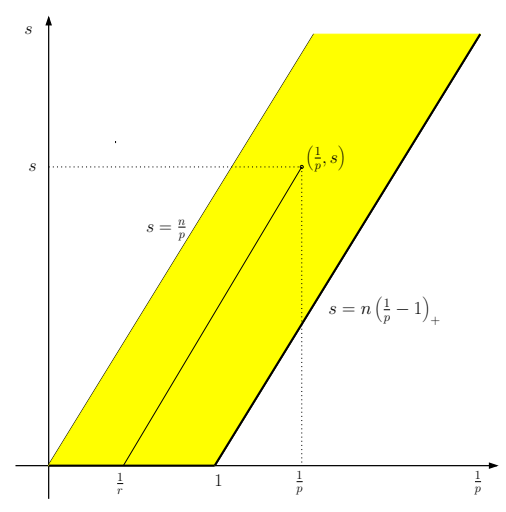

FiguRE 1. Sub-critical case

Proposition 4.1. Let $\alpha$ be an anisotropy according to (2). Furthermore let $s, p$ and $q$ be as in (20) and define $r \in(1, \infty)$ by the equation

$$
s-\frac{n}{p}=-\frac{n}{r} \text {. }
$$

Then there is a constant $c>0$ such that

$$
\mathcal{E}_{\mathrm{G}} B_{p q}^{s, \alpha}\left(\mathbb{R}^{n}\right)(t) \leq c t^{-\frac{1}{r}} \quad \text { for } \quad t \in(0,1]
$$

Moreover, for each $v \in[q, \infty]$ there is a positive constant $c$, depending only on $v$, such that

$$
\left(\int_{0}^{1}\left(t^{1 / r} f^{*}(t)\right)^{v} \frac{\mathrm{d} t}{t}\right)^{1 / v} \leq c\left\|f \mid B_{p q}^{s, \alpha}\left(\mathbb{R}^{n}\right)\right\| \text { for all } f \in B_{p q}^{s, \alpha}\left(\mathbb{R}^{n}\right),
$$

with the modification as in (1) on the left-hand side for $v=\infty$.

Proof: Our method will be an adaptation of the reasoning used on pp. 189191 of [17], but we have to examine very carefully the influence of the anisotropy.

As $(1 / p, s)$ belongs to the subcritical strip (see Figure 1 ), there are $s_{0}, s_{1} \in$ $\mathbb{R}$ such that $s_{0}<s<s_{1}$ and $\left(1 / p, s_{i}\right), i=0,1$, also belong to the subcritical strip. Define $r_{i}$ by $s_{i}-n / p=-n / r_{i}, i=0,1$. Plainly $r_{i} \in(1, \infty)$ and by elementary embeddings, Proposition 2.5 and Proposition 2.3 we obtain

$$
B_{p 1}^{s_{i}, \alpha}\left(\mathbb{R}^{n}\right) \hookrightarrow B_{p r_{i}}^{s_{i}, \alpha}\left(\mathbb{R}^{n}\right) \hookrightarrow F_{r_{i} 2}^{0, \alpha}\left(\mathbb{R}^{n}\right)=L_{r_{i}} \quad \text { for } i=0,1 .
$$

We complete the proof by using the real interpolation method. By virtue of Proposition 2.4, with $0<\theta<1$ and $s=(1-\theta) s_{0}+\theta s_{1}$, we have

$$
\left(B_{p 1}^{s_{0}, \alpha}\left(\mathbb{R}^{n}\right), B_{p 1}^{s_{1}, \alpha}\left(\mathbb{R}^{n}\right)\right)_{\theta, v}=B_{p v}^{s, \alpha}\left(\mathbb{R}^{n}\right)
$$


for any $0<v \leq \infty$. Since $1<r_{0}<r_{1}<\infty$ and $1 / r=(1-\theta) / r_{0}+\theta / r_{1}$, we obtain

$$
\left(L_{r_{0}}, L_{r_{1}}\right)_{\theta, v}=L_{r v}
$$

Thus the interpolation property yields,

$$
B_{p v}^{s, \alpha}\left(\mathbb{R}^{n}\right)=\left(B_{p 1}^{s_{0}, \alpha}\left(\mathbb{R}^{n}\right), B_{p 1}^{s_{1}, \alpha}\left(\mathbb{R}^{n}\right)\right)_{\theta, v} \hookrightarrow\left(L_{r_{0}}, L_{r_{1}}\right)_{\theta, v}=L_{r, v} .
$$

Translating the above embedding into the language of inequalities, there is a constant $c>0$ such that

$$
\left(\int_{0}^{1}\left(t^{1 / r} f^{*}(t)\right)^{v} \frac{\mathrm{d} t}{t}\right)^{1 / v} \leq c\left\|f \mid B_{p v}^{s, \alpha}\left(\mathbb{R}^{n}\right)\right\| \quad \text { for all } f \in B_{p v}^{s, \alpha}\left(\mathbb{R}^{n}\right) .
$$

Taking into account that $v \geq q$ we have, by elementary embeddings, that $\left\|f\left|B_{p v}^{s, \alpha}\left(\mathbb{R}^{n}\right)\|\leq c\| f\right| B_{p q}^{s, \alpha}\left(\mathbb{R}^{n}\right)\right\|$, which completes the proof of (23). To establish (22) we now use the already proved inequality (23) with $v=\infty$, to obtain

$$
\sup _{0<t<\varepsilon} t^{\frac{1}{r}} f^{*}(t) \leq c\left\|f \mid B_{p q}^{s, \alpha}\left(\mathbb{R}^{n}\right)\right\| .
$$

This, together with (12), finishes the proof.

Proposition 4.2. Let $\alpha, p, q, s$ and $r$ be as in Proposition 4.1. Then there are an $\varepsilon \in(0,1)$ and a constant $c>0$ such that

$$
\mathcal{E}_{\mathrm{G}} B_{p q}^{s, \alpha}\left(\mathbb{R}^{n}\right)(t) \geq c t^{-\frac{1}{r}} \quad \text { for all } \quad t \in(0, \varepsilon] .
$$

Moreover, for each $v \in(0, q)$ there is no positive constant $c$ for which

$$
\left(\int_{0}^{\varepsilon}\left(t^{1 / r} f^{*}(t)\right)^{v} \frac{\mathrm{d} t}{t}\right)^{1 / v} \leq c\left\|f \mid B_{p q}^{s, \alpha}\left(\mathbb{R}^{n}\right)\right\|
$$

holds for all $f \in B_{p q}^{s, \alpha}\left(\mathbb{R}^{n}\right)$.

Proof: Our proof is based upon ideas found in Steps 2 and 3 in the proof of $[17$, Theorem 15.2] with necessary modification which comes from the influence of the anisotropy. Let us consider the function $\varphi$ given by

$$
\varphi(x):=\left\{\begin{array}{lll}
\exp \left(-\frac{1}{1-|x|^{2}}\right) & \text { for } & |x|<1 \\
0 & \text { for } & |x| \geq 1
\end{array}\right.
$$


and define

$$
f_{j}(x):=2^{j n / r} \varphi\left(2^{j \alpha} x\right), \quad x \in \mathbb{R}^{n}, \quad j \in \mathbb{N} .
$$

It turns out that the $f_{j}$ 's are, up to an unimportant constant, atoms in $B_{p q}^{s, \alpha}\left(\mathbb{R}^{n}\right)$ according to the Definition 2.6 when considering the subcritical case. We first note that no moment conditions are needed. Let us check the remaining conditions from the definition of atoms. For $j \in \mathbb{N}$, we have

$$
\begin{aligned}
\operatorname{supp} f_{j} \subset\left\{x \in \mathbb{R}^{n}:\left|2^{j \alpha} x\right| \leq 1\right\} & \subset\left\{x \in \mathbb{R}^{n}:\left|x_{k}\right| \leq 2^{-j \alpha_{k}}, k=1, \ldots, n\right\} \\
& \subset c Q_{j 0}^{\alpha}
\end{aligned}
$$

and

$$
\left|\mathrm{D}^{\gamma} f_{j}(x)\right|=2^{j n / r}\left|\mathrm{D}^{\gamma} \varphi\left(2^{j \alpha} x\right)\right| \leq \sup _{|y| \leq 1} \sup _{\alpha \gamma \leq K}\left|\mathrm{D}^{\gamma} \varphi(y)\right| 2^{-\alpha \gamma} 2^{j n / r} \leq c 2^{-j\left(s-\frac{n}{p}-\gamma \alpha\right)} .
$$

For the distribution function of $f_{j}$ we obtain

$$
\begin{aligned}
\mu_{f_{j}}(\lambda) & =\left|\left\{x \in \mathbb{R}^{n}: \quad\left|f_{j}(x)\right|>\lambda\right\}\right|=\left|\left\{x \in \mathbb{R}^{n}: \quad\left|\varphi\left(2^{j \alpha} x\right)\right|>2^{-j n / r} \lambda\right\}\right| \\
& =\left|\left\{2^{-j \alpha} y: \quad|\varphi(y)|>2^{-j n / r} \lambda\right\}\right|=2^{-j \alpha} \mu_{\varphi}\left(2^{-j n / r} \lambda\right), \quad j \in \mathbb{N} .
\end{aligned}
$$

Note that in the last equality we have used the fact that $\sum_{j=1}^{n} \alpha_{j}=n$. Consequently,

$$
f_{j}^{*}\left(d 2^{-j n}\right) \sim 2^{j n / r}, \quad j \in \mathbb{N},
$$

for some $d>0$, and therefore

$$
\mathcal{E}_{\mathrm{G}} B_{p q}^{s, \alpha}\left(\mathbb{R}^{n}\right)\left(d 2^{-j n}\right) \geq c f_{j}^{*}\left(d 2^{-j n}\right) \sim 2^{j n / r}, \quad j \in \mathbb{N} .
$$

Then the monotonicity of the growth envelope function together with its definition proves $(25)$.

The next step of the proof is to show the sharpness expressed by the second assertion of our proposition.

Suppose that, contrary to our claim, there exist a $v<q$ and a constant $c>0$ such that

$$
\left(\int_{0}^{\varepsilon}\left(t^{1 / r} f^{*}(t)\right)^{v} \frac{\mathrm{d} t}{t}\right)^{1 / v} \leq c\left\|f \mid B_{p q}^{s, \alpha}\left(\mathbb{R}^{n}\right)\right\|
$$

holds for all $f \in B_{p q}^{s, \alpha}\left(\mathbb{R}^{n}\right)$. 
Consider, for each $J \in \mathbb{N}, f \in B_{p q}^{s, \alpha}\left(\mathbb{R}^{n}\right)$ defined by

$$
f(x)=\sum_{j=1}^{J} 2^{j n / r} \varphi\left(2^{j \alpha} x-x^{0}\right)
$$

where $\varphi$ has the same meaning as before and $x^{0} \in \mathbb{Z}^{n}$ is chosen in such a way that the supports of $\varphi\left(2^{j \alpha} \cdot-x^{0}\right), j \in \mathbb{N}$, are disjoint. Note that it is sufficient to take $x^{0}=\left(x_{1}^{0}, \ldots, x_{n}^{0}\right) \in \mathbb{R}^{n}$ such that

$$
\left|x_{k}^{0}\right|>1+\frac{2}{2^{\alpha_{k}}-1}, \quad k=1, \ldots, n .
$$

Then it follows that

$$
f^{*}\left(d 2^{-j n}\right) \geq c 2^{j n / r}, \quad j=1, \ldots, J
$$

with some $c, d>0$. Plugging (31) into (29) and using an equivalent quasinorm (11) coming from atomic decomposition as stated in Theorem 2.8, we obtain

$$
\left(\sum_{j=1}^{J} 1\right)^{1 / v} \leq c_{1}\left\|f \mid B_{p q}^{s, \alpha}\left(\mathbb{R}^{n}\right)\right\| \leq c_{2}\left(\sum_{j=1}^{J} 1\right)^{1 / q},
$$

where the constants $c_{1}, c_{2}>0$ are independent of $J$. This is a clear contradiction to the fact that $v<q$, which completes the proof.

It is now easy to see that by virtue of (22) jointly with (25) we immediately obtain $\mathcal{E}_{\mathrm{G}} B_{p q}^{s, \alpha}(t) \sim t^{-1 / r}$. Moreover, the second part of Proposition 4.2 shows that $q$ is the optimal index. This already gives the proof for the first part of the following theorem.

Theorem 4.3. Let $\alpha, p, q, s$ and $r$ be as in Proposition 4.1. Then

(i) $\mathfrak{E}_{\mathrm{G}} B_{p q}^{s, \alpha}\left(\mathbb{R}^{n}\right)=\left(t^{-\frac{1}{r}}, q\right)$;

(ii) $\mathfrak{E}_{\mathrm{G}} F_{p q}^{s, \alpha}\left(\mathbb{R}^{n}\right)=\left(t^{-\frac{1}{r}}, p\right)$.

Proof: It only remains to verify (ii). Let $s_{0}, s_{1} \in \mathbb{R}$ and $0<p_{0}<p<p_{1} \leq \infty$ be such that $\left(1 / p_{i}, s_{i}\right), i=0,1$, belong to the subcritical strip, i.e. $\sigma_{p_{i}}<s_{i}<$ $n / p_{i}, i=0,1$, and $s_{0}-n / p_{0}=s-n / p=s_{1}-n / p_{1}=-n / r$. Then, by Proposition 2.5, we have

$$
B_{p_{1} p}^{s_{1}, \alpha}\left(\mathbb{R}^{n}\right) \hookrightarrow F_{p q}^{s, \alpha}\left(\mathbb{R}^{n}\right) \hookrightarrow B_{p_{0} p}^{s_{0}, \alpha}\left(\mathbb{R}^{n}\right) .
$$


Then, by part (i) and Proposition 2.10(ii), we obtain

$$
t^{-1 / r} \sim \mathcal{E}_{\mathrm{G}} B_{p_{1} q}^{s_{1}, \alpha}(t) \lesssim \mathcal{E}_{\mathrm{G}} F_{p q}^{s, \alpha}(t) \lesssim \mathcal{E}_{\mathrm{G}} B_{p_{0} q}^{s_{0}, \alpha}(t) \sim t^{-1 / r}, \quad t \in(0, \varepsilon),
$$

for some $\varepsilon \in(0,1)$. This fact, together with (33), Proposition 2.10 (iii) and part (i) of the present theorem, leads us to

$$
p=u_{B_{p_{1} p}^{s_{1}, \alpha}\left(\mathbb{R}^{n}\right)} \leq u_{F_{p q}^{s, \alpha}\left(\mathbb{R}^{n}\right)} \leq u_{B_{p_{0} p}^{s_{0}, \alpha}\left(\mathbb{R}^{n}\right)}=p
$$

which finishes the proof.

\section{Growth envelopes of anisotropic function spaces in the critical case}

In this section we consider the spaces $A_{p q}^{s, \alpha}\left(\mathbb{R}^{n}\right)$ in the critical case, which means that the corresponding parameters satisfy

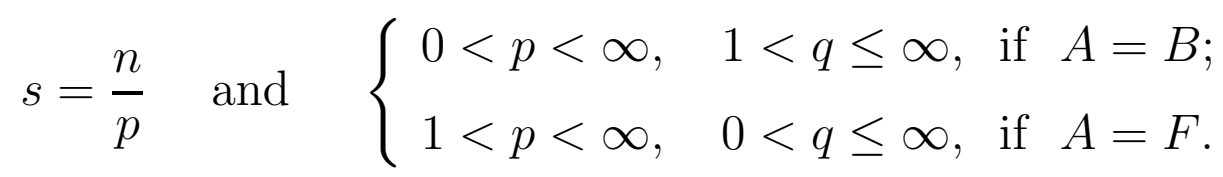

According to Proposition 3.2, the embedding $A_{p q}^{s, \alpha}\left(\mathbb{R}^{n}\right) \hookrightarrow L_{\infty}\left(\mathbb{R}^{n}\right)$, in the critical case, does not hold.

Theorem 5.1. Let $\alpha$ be an anisotropy according to (2).

(i) Let $0<p \leq \infty$ and $1<q \leq \infty$. Then

$$
\mathfrak{E}_{\mathrm{G}} B_{p q}^{n / p, \alpha}\left(\mathbb{R}^{n}\right)=\left(|\log t|^{1 / q^{\prime}}, q\right) .
$$

(ii) Let $1<p<\infty$ and $0<q \leq \infty$. Then

$$
\mathfrak{E}_{\mathrm{G}} F_{p q}^{n / p, \alpha}\left(\mathbb{R}^{n}\right)=\left(|\log t|^{1 / p^{\prime}}, p\right) .
$$

Proof: Although our proof runs along similar lines as the proof of Theorem 13.2 of [17], there are subtle adjustments necessary to fit the argument to the anisotropic situation. We divide the proof into 3 steps. In the first two steps we compute the growth envelope function in $B$ and $F$-case. Hereby we closely follow the argument given in [10]. The last step contains the proof of the correctness of corresponding indices.

Step 1. Let us work with $p$ and $q$ as assumed in (i). The first objective is to prove that there exists a constant $c>0$ such that

$$
\left(\int_{0}^{\varepsilon}\left(\frac{f^{*}(t)}{|\log t|}\right)^{q} \frac{\mathrm{d} t}{t}\right)^{1 / q} \leq c\left\|f \mid B_{p q}^{n / p, \alpha}\left(\mathbb{R}^{n}\right)\right\| \text { for all } f \in B_{p q}^{n / p, \alpha}\left(\mathbb{R}^{n}\right)
$$


with the obvious modification in the case $q=\infty$. Remark that by virtue of elementary embeddings, there is no loss of generality in assuming that $1<p<\infty$ and $1<q \leq \infty$. Let us consider an optimal atomic decomposition of a given $f \in B_{p q}^{n / p, \alpha}\left(\mathbb{R}^{n}\right)$ in the form

$$
f=\sum_{\nu=0}^{\infty} f_{\nu} \quad \text { with } \quad f_{\nu}(x)=\sum_{m \in \mathbb{Z}^{n}} b_{\nu m} a_{\nu m}^{\alpha}(x)
$$

Additionally, the formula

$$
\left(\sum_{\nu=0}^{\infty}\left(\sum_{m \in \mathbb{Z}^{n}}\left|b_{\nu m}\right|^{p}\right)^{q / p}\right)^{1 / p}
$$

gives an equivalent quasi-norm on $B_{p q}^{n / p, \alpha}$. It may be worth reminding that $a_{\nu, m}^{\alpha}$ have the following properties

- $\operatorname{supp} a_{\nu m}^{\alpha} \subset\left\{y \in \mathbb{R}^{n}:\left|y_{k}-2^{-\nu \alpha_{k}} m_{k}\right|<d 2^{-\nu \alpha_{k}}, k=1, \ldots, n\right\} \quad$ for some $d>1, \nu \in \mathbb{N}_{0}, m \in \mathbb{Z}^{n}$,

- $\left|\mathrm{D}^{\gamma} a_{\nu m}^{\alpha}(x)\right| \leq 2^{\nu \gamma \alpha}$ for $\alpha \gamma \leq K, x \in \mathbb{R}^{n}$.

Let us now denote by $\chi_{\nu l}(t)$ the characteristic function of the interval $\left(C 2^{-\nu n}(l-\right.$ 1), $\left.C 2^{-\nu n} l\right]$ with $C>0$ to be computed later, $\nu \in \mathbb{N}_{0}$ and $l \in \mathbb{N}$. Furthermore for fixed $\nu \in \mathbb{N}_{0}$ let $\left(b_{\nu l}^{*}\right)_{l \in \mathbb{N}}$ stand for the non-increasing rearrangement of $\left(b_{\nu m}\right)_{m \in \mathbb{Z}^{n}}$. We now prove that

$$
f_{\nu}^{*}(t) \leq c \sum_{l=1}^{\infty} b_{\nu l}^{*} \chi_{\nu m}(t), \quad \text { with } t>0 \text { and } \nu \in \mathbb{N}_{0}
$$

Let $\in \mathbb{N}_{0}$ and $D:=(4 d)^{n}$. Then

$$
\left(D^{-1} f_{\nu}\right)^{*}(t)=\inf \left\{\lambda>0:\left|\left\{x \in \mathbb{R}^{n}:\left|D^{-1} \sum_{m \in \mathbb{Z}^{n}} b_{\nu m} a_{\nu m}^{\alpha}(x)\right|>\lambda\right\}\right| \leq t\right\} .
$$


Suppose that $t \in\left(C 2^{-\nu n}(l-1), C 2^{-\nu n} l\right]$ for some $l \in \mathbb{N}$ and $C>0$ to be chosen later. We remark that if $x \in \mathbb{R}^{n}$ is such that

$$
\begin{aligned}
b_{\nu l}^{*} & \leq D^{-1}\left|\sum_{m \in \mathbb{Z}^{n}} b_{\nu m} a_{\nu m}^{\alpha}(x)\right| \\
& \leq D^{-1} \sum_{m \in \mathbb{Z}^{n}}\left|b_{\nu m}\right|\left|a_{\nu m}^{\alpha}(x)\right| \chi_{2 d Q_{\nu m}^{\alpha}}(x) \\
& \leq D^{-1} \sum_{m \in \mathbb{Z}^{n}}\left|b_{\nu m}\right| \chi_{2 d Q_{\nu m}^{\alpha}}(x)
\end{aligned}
$$

then, for such an $x, b_{\nu l}^{*}<\left|b_{\nu m^{\prime}}\right|$, where $m^{\prime}$ is such that $\left|b_{\nu m^{\prime}}\right|$ is the biggest from all $D$ possibilities of $x \in 2 d Q_{\nu m}^{\alpha}$. Hence $x \in 2 d Q_{\nu m}^{\alpha}$ such that $b_{\nu l}^{*}<\left|b_{\nu m}\right|$. By definition of $b_{\nu l}^{*}$, for any $k \in \mathbb{N}$ the number of $m$ 's such that $\left|b_{\nu m}\right| \geq b_{\nu l}^{*}+1 / k$ is less or equal to $l-1$. Therefore, we get

$$
\begin{aligned}
& \left|\left\{x \in \mathbb{R}^{n}:\left|D^{-1} \sum_{m \in \mathbb{Z}^{n}} b_{\nu m} a_{\nu m}^{\alpha}(x)\right|>b_{\nu l}^{*}\right\}\right| \\
& =\left|\bigcap_{k \in \mathbb{N}}\left\{x \in \mathbb{R}^{n}:\left|D^{-1} \sum_{m \in \mathbb{Z}^{n}} b_{\nu m} a_{\nu m}^{\alpha}(x)\right|>b_{\nu l}^{*}+\frac{1}{k}\right\}\right| \\
& =\lim _{k \rightarrow \infty}\left|\left\{x \in \mathbb{R}^{n}:\left|D^{-1} \sum_{m \in \mathbb{Z}^{n}} b_{\nu m} a_{\nu m}^{\alpha}(x)\right|>b_{\nu l}^{*}+\frac{1}{k}\right\}\right| \\
& \leq \lim _{k \rightarrow \infty}\left|\bigcup_{\left\{m:\left|b_{\nu m}\right| \geq b_{\nu l}^{*}+1 / k\right\}} 2 d Q_{\nu m}^{\alpha}\right| \leq \lim _{k \rightarrow \infty} \sum_{\left\{m:\left|b_{\nu m}\right| \geq b_{\nu l}^{*}+1 / k\right\}}\left|2 d Q_{\nu m}^{\alpha}\right| \\
& \leq(l-1) 2^{n+1} d^{n} 2^{-\nu n}<t .
\end{aligned}
$$

Taking $C:=2^{n+1} d^{n}$ we arrive at

$$
f_{\nu}^{*}(t) \leq D b_{\nu l}^{*} \quad \text { for } \quad t \in\left(C 2^{-\nu n}(l-1), C 2^{-\nu n} l\right],
$$

which proves (39). The rest of the proof of (37) is an exact analogue of the proof of Theorem 13.2 of [17]. For a detailed exposition we refer the reader to this monograph. Let us only mention that it relies on a clever application of the Hardy-Littlewood maximal inequality. By virtue of (37) we immediately obtain

$$
\sup _{0<t<\varepsilon} \frac{f^{*}(t)}{|\log t|^{1 / q^{\prime}}} \leq c\left\|f \mid B_{p q}^{\frac{n}{p}, \alpha}\left(\mathbb{R}^{n}\right)\right\|
$$


which shows that

$$
\mathcal{E}_{\mathrm{G}} B_{p q}^{\frac{n}{p}, \alpha}(t) \leq c|\log t|^{1 / q^{\prime}} \quad \text { for } \quad 0<p<\infty, \quad 1<q \leq \infty .
$$

To prove the $F$-result, we again exploit embeddings described in Proposition 2.5 jointly with the properties of growth envelope functions to get

$$
\mathcal{E}_{\mathrm{G}} F_{p q}^{\frac{n}{p}, \alpha}(t) \leq c|\log t|^{1 / p^{\prime}} \quad \text { for } \quad 1<p<\infty, \quad 0<q \leq \infty .
$$

Step 2. We shall prove the converse of the inequality (40), that is

$$
\mathcal{E}_{\mathrm{G}} B_{p q}^{\frac{n}{p}, \alpha}(t) \geq c|\log t|^{1 / q^{\prime}} \quad \text { for } \quad 0<p<\infty, \quad 1<q \leq \infty .
$$

To see this, we again benefit from the construction of extremal functions (see [17] for the isotropic case). Let us consider

$$
f(x):=\sum_{j=1}^{\infty} b_{j} \varphi\left(2^{(j-1) \alpha} x\right), \quad x \in \mathbb{R}^{n}
$$

where the function $\varphi$ is given by $(27)$ and $b=\left(b_{j}\right)_{j=1}^{\infty}$ is a sequence of positive numbers with $b \in \ell_{q}$. In a similar way as in the subcritical case we may check that $a_{j}(x):=\varphi\left(2^{(j-1) \alpha} x\right)$ with $x \in \mathbb{R}^{n}$ and $j \in \mathbb{N}$ are, up to constants, $(n / p, p)_{K, 0}^{\alpha}$-atoms. Next, let $x \in \mathbb{R}^{n}$ be such that $1 / 4 \leq\left|2^{(k-1) \alpha} x\right| \leq 1 / 2$ for some $k \in \mathbb{N}$. Easy computation shows that putting

$$
k_{0}(\alpha)=\left\{\begin{array}{lrr}
2 \alpha_{1}^{-1}-1 & \text { for } & 2 \alpha_{1}^{-1} \in \mathbb{N} \\
{\left[2 \alpha_{1}^{-1}\right]} & \text { otherwise }
\end{array}\right.
$$

yields

$$
f(x)=\sum_{j=1}^{k+k_{0}(\alpha)} b_{j} \varphi\left(2^{(j-1) \alpha} x\right) .
$$

Hence we immediately obtain

$$
f(x) \leq e^{-1} \sum_{j=1}^{k+k_{0}(\alpha)} b_{j} \quad \text { and } \quad f(x) \geq e^{-4 / 3} \sum_{j=1}^{k} b_{j} .
$$


Consequently, for $\lambda$ with $0<\lambda<c \sum_{j=1}^{k} b_{j}$, where $c=e^{-4 / 3}$ we get

$$
\begin{aligned}
\mu_{f}(\lambda) & \geq\left|\left\{x \in \mathbb{R}^{n}: \quad|f(x)| \geq c \sum_{j=1}^{k} b_{j}\right\}\right| \\
& \geq\left|\left\{x \in \mathbb{R}^{n}: \quad 1 / 4 \leq\left|2^{(k-1) \alpha} x\right| \leq 1 / 2\right\}\right| \\
& =2^{-(k-1) n}|\{y: \quad 1 / 4 \leq|y| \leq 1 / 2\}|=c^{\prime} 2^{-k n} .
\end{aligned}
$$

Therefore it follows that, for $0<t<c^{\prime} 2^{-k n}$,

$$
f^{*}(t) \geq c \sum_{j=1}^{k} b_{j} .
$$

For each $J \in \mathbb{N}$ we consider the function $f=f_{J}$ defined by (42) with the special sequence $b=\left(b_{j}\right)_{j=1}^{\infty}$ given by

$$
b_{j}:=\left\{\begin{array}{lll}
J^{-1 / q} & \text { for } & j=1, \ldots J \\
0 & & \text { otherwise. }
\end{array}\right.
$$

It is easy to see that $\left\|b \mid \ell_{q}\right\|=1$. Thus, (43) yields

$$
f_{J}^{*}\left(c^{\prime} 2^{-(J+1) n}\right) \geq c \sum_{j=1}^{J} b_{j}=c J^{-1 / q^{\prime}} .
$$

Put $c^{\prime} 2^{-(J+1) n} \leq t \leq c^{\prime} 2^{-J n}$ for some $J \in \mathbb{N}$. Then, using monotonicity of the growth envelope function $\mathcal{E}_{\mathrm{G}} B_{p q}^{\frac{n}{p}, \alpha}(\cdot)$ together with (44) yields

$$
\mathcal{E}_{\mathrm{G}} B_{p q}^{\frac{n}{p}, \alpha}(t) \geq \mathcal{E}_{\mathrm{G}} B_{p q}^{\frac{n}{p}, \alpha}\left(2^{-(J+1) n}\right) \geq c f_{J}^{*}\left(2^{-(J+1) n}\right) \geq c J^{1 / q} \geq c|\log t|^{1 / q^{\prime}} .
$$

The $F$-counterpart follows from the embedding $B_{r p}^{n / p, \alpha}\left(\mathbb{R}^{n}\right) \hookrightarrow F_{p q}^{n / p, \alpha}\left(\mathbb{R}^{n}\right)$ with $0<r<p$, since then

$$
\mathcal{E}_{\mathrm{G}} F_{p q}^{\frac{n}{p}, \alpha}(t) \geq \mathcal{E}_{\mathrm{G}} B_{r, p}^{\frac{n}{p}, \alpha}(t) \geq c|\log t|^{1 / p^{\prime}} .
$$

Step 3. In the reminder of the proof we show that the indices $q$ in the $B$-case and $p$ in the $F$-case cannot be improved. The proof of this part for $B$ spaces is essentially a repetition of the arguments used to prove the isotropic case. For the sake of completeness we repeat the main steps of this proof. 
We proceed by assuming that there exist a $v<q$ and a constant $c>0$ such that

$$
\left(\int_{0}^{\varepsilon}\left(\frac{f^{*}(t)}{|\log t|^{1 / q^{\prime}}}\right)^{v} \frac{\mathrm{d} t}{t|\log t|}\right)^{1 / v} \leq c\left\|f \mid B_{p q}^{n / p, \alpha}\left(\mathbb{R}^{n}\right)\right\|
$$

holds for all $f \in B_{p q}^{n / p, \alpha}\left(\mathbb{R}^{n}\right)$. This stands in contrary to our claim. We again consider the function $f$ given by $(42)$, but this time we work with the sequence $b=\left(b_{j}\right)_{j=1}^{\infty}$ defined by

$$
b_{j}:= \begin{cases}0 & \text { for } \quad j=1 \\ j^{-1 / q}(\log (j))^{-1 / v} & \text { for } \quad j \geq 2 .\end{cases}
$$

Note that $b \in \ell_{q}$ and hence the right-hand side of (47) is bounded. Plugging the inequality (43) into the left-hand side of (47) we may estimate it from below by

$$
c_{1} \sum_{k=M}^{\infty}\left(k b_{k}\right)^{v} k^{-v / q^{\prime}-1}=c_{1} \sum_{k=M}^{\infty} k^{-1}(\log k)^{-1}=\infty,
$$

which gives a contradiction. The theorem is proved, since the correctness of the exponent for the $F$-case follows as in the subcritical case.

Remark 5.2. The necessity of the conditions in Proposition 3.2 are a consequence of the last theorem, due to Proposition 2.10(i).

\section{Anisotropic Hardy inequalities}

This section deals with inequalities of Hardy type related to the anisotropic function spaces $B_{p q}^{s, \alpha}\left(\mathbb{R}^{n}\right)$ and $F_{p q}^{s, \alpha}\left(\mathbb{R}^{n}\right)$. Hardy inequalities related to the isotropic function spaces of Besov and Triebel-Lizorkin type have been studied in [17, Section 16]. The results we present in this section are essentially adaptations of reasoning used there to the anisotropic case. In the following applications use will be made of envelopes results obtained in last two sections.

In the sequel, if $\varepsilon>0$ then $K_{\varepsilon}^{\alpha}:=\left\{x \in \mathbb{R}^{n}:|x|_{\alpha}<\varepsilon\right\}$ is the anisotropic ball centered at the origin with (anisotropic) radius $\varepsilon$. The Lebesgue measure of such a ball is $\left|K_{\varepsilon}^{\alpha}\right|=c \varepsilon^{n}$ with $c$ independent of $\varepsilon$.

Theorem 6.1. Let $\varepsilon>0$ and let $\varkappa$ be a positive monotonically decreasing function on $(0, \varepsilon]$. Let $\alpha$ be an anisotropy according to (2), $s>0$ and $s-$ $n / p=-n / r$ with $1<r<\infty$. 
(i) Let $0<q \leq r$. Then

$$
\left.\left.\int_{K_{\varepsilon}^{\alpha}}\left|\varkappa\left(|x|_{\alpha}\right)\right| x\right|_{\alpha} ^{n / r} f(x)\right|^{q} \frac{\mathrm{d} x}{|x|_{\alpha}^{n}} \leq c\left\|f \mid B_{p q}^{s, \alpha}\left(\mathbb{R}^{n}\right)\right\|^{q}
$$

for some $c>0$ and all $f \in B_{p q}^{s, \alpha}\left(\mathbb{R}^{n}\right)$ if, and only if, $\varkappa$ is bounded.

(ii) Let $0<q \leq \infty$. Then

$$
\left.\left.\int_{K_{\varepsilon}^{\alpha}}\left|\varkappa\left(|x|_{\alpha}\right)\right| x\right|_{\alpha} ^{n / r} f(x)\right|^{p} \frac{\mathrm{d} x}{|x|_{\alpha}^{n}} \leq c\left\|f \mid F_{p q}^{s, \alpha}\left(\mathbb{R}^{n}\right)\right\|^{p}
$$

for some $c>0$ and all $f \in F_{p q}^{s, \alpha}\left(\mathbb{R}^{n}\right)$ if, and only if, $\varkappa$ is bounded.

Proof: We begin by proving (48) with $\varkappa=1$. Let $c>0$ and consider

$$
b(x):=\left\{\begin{array}{lll}
|x|_{\alpha}^{-c n} & \text { for } & x \in \overline{K_{\varepsilon}^{\alpha}} \\
0 & \text { for } & x \notin K_{\varepsilon}^{\alpha} .
\end{array}\right.
$$

Then $b^{*}(t) \sim t^{-c}$ near the origin. We now apply this observation with $c=$ $1 / q-1 / r$ to estimate

$$
\begin{aligned}
& \left.\left.\int_{K_{\varepsilon}^{\alpha}}|| x\right|_{\alpha} ^{n / r} f(x)\right|^{q} \frac{\mathrm{d} x}{|x|_{\alpha}^{n}}=\int_{K_{\varepsilon}^{\alpha}} b^{q}(x)|f(x)|^{q} \mathrm{~d} x \\
& \leq \int_{0}^{\left|K_{\varepsilon}^{\alpha}\right|} b^{* q}(t) f^{* q}(t) \mathrm{d} t \sim \int_{0}^{c_{1} \varepsilon^{n}}\left(t^{1 / r} f^{*}(t)\right)^{q} \frac{\mathrm{d} t}{t} \leq c_{2}\left\|f \mid B_{p q}^{s, \alpha}\left(\mathbb{R}^{n}\right)\right\|^{q} .
\end{aligned}
$$

For the first inequality we have used a well-known property (see $[1$, Chapter II, Corollary 4.5]). The second inequality follows from Proposition 4.1. The proof of (i) is completed by showing that $\varkappa$ has to be bounded if (48) holds. For simplicity, let us assume that $\varepsilon=1$. Suppose that, contrary to our claim, $\varkappa(t) \rightarrow \infty$ as $t \downarrow 0$. The argument here makes essential use of the extremal function given by (28). For more details we refer the reader to the proof of Proposition 4.2. After plugging these functions into (48), we conclude that $\varkappa\left(2^{-j}\right) \leq c$, for any sufficiently large $j$, which is a contradiction.

The proof of (ii) can be handled in much the same way as in the proof of Theorem 4.3 (ii), namely by using Proposition 2.5. This method enable us to avoid the use of extremal functions in $F$-spaces that might require moment conditions, as was done in [17, Step 5 in the proof of Theorem 13.2] for the isotropic case.

Theorem 6.2. Let $\varepsilon \in(0,1)$ and let $\varkappa$ be a positive monotonically decreasing function on $(0, \varepsilon]$. Moreover, let $\alpha$ be an anisotropy according to (2). 
(i) Let $0<p<\infty$ and $1<q<\infty$. Then

$$
\int_{K_{\varepsilon}^{\alpha}}\left|\frac{\varkappa\left(|x|_{\alpha}\right) f(x)}{\log |x|_{\alpha}}\right|^{q} \frac{\mathrm{d} x}{|x|_{\alpha}^{n}} \leq c\left\|f \mid B_{p q}^{n / p, \alpha}\left(\mathbb{R}^{n}\right)\right\|^{q}
$$

for some $c>0$ and all $f \in B_{p q}^{n / p, \alpha}\left(\mathbb{R}^{n}\right)$ if, and only if, $\varkappa$ is bounded.

(ii) Let $1<p<\infty$ and $0<q \leq \infty$. Then

$$
\int_{K_{\varepsilon}^{\alpha}}\left|\frac{\varkappa\left(|x|_{\alpha}\right) f(x)}{\log |x|_{\alpha}}\right|^{p} \frac{\mathrm{d} x}{|x|_{\alpha}^{n}} \leq c\left\|f \mid F_{p q}^{n / p, \alpha}\left(\mathbb{R}^{n}\right)\right\|^{p}
$$

for some $c>0$ and all $f \in F_{p q}^{n / p, \alpha}\left(\mathbb{R}^{n}\right)$ if, and only if, $\varkappa$ is bounded.

Proof: Let us first prove (50) with $\varkappa=1$. We consider

$$
a(x):=\left\{\begin{array}{lll}
\left.\left.|x|_{\alpha}^{-n / q}|\log | x\right|_{\alpha}\right|^{-1} & \text { for } & x \in \overline{K_{\varepsilon}^{\alpha}} \\
0 & \text { for } & x \notin K_{\varepsilon}^{\alpha} .
\end{array}\right.
$$

Computing the measure-preserving rearrangement of $a$ yields $a^{*}(t) \sim t^{-1 / q}|\log t|^{-1}$ for $t$ near the origin. To prove this we have used asymptotic inversion, in particular the assertions of Proposition 1.1.15 and Appendix 5/2. of [2]. Hence,

$$
\begin{aligned}
& \int_{K_{\varepsilon}^{\alpha}}\left|\frac{f(x)}{\log |x|_{\alpha}}\right|^{q} \frac{\mathrm{d} x}{|x|_{\alpha}^{n}}=\int_{K_{\varepsilon}^{\alpha}} a^{q}(x)|f(x)|^{q} \mathrm{~d} x \\
& \leq \int_{0}^{\left|K_{\varepsilon}^{\alpha}\right|} a^{* q}(t) f^{* q}(t) \mathrm{d} t \sim \int_{0}^{c_{1} \varepsilon^{n}}\left|\frac{f^{*}(t)}{\log t}\right|^{q} \frac{\mathrm{d} t}{t} \leq c_{2}\left\|f \mid B_{p q}^{n / p, \alpha}\left(\mathbb{R}^{n}\right)\right\|^{q},
\end{aligned}
$$

which gives (50). Once again, for the first inequality we have used a wellknown property of rearrangements. The second inequality follows from Proposition 5.1 (i). For the reverse implication we must show that $\varkappa$ is bounded. Again for simplicity, let us assume that $\varepsilon=1$. To obtain a contradiction, we suppose that $\varkappa(t) \rightarrow \infty$ as $t \downarrow 0$. Moreover, assume that $f(x)$ is a positive monotonically decreasing function in $K_{1}^{\alpha}$. By virtue of monotonicity of $\varkappa$ we get

$$
\begin{aligned}
\sup _{0<t<\delta} & \frac{\varkappa(t) f^{*}(t)}{|\log t|^{1 / q^{\prime}}} \leq c_{1}\left(\int_{0}^{\delta}\left(\frac{\varkappa(t) f^{*}(t)}{|\log t|}\right)^{q} \frac{\mathrm{d} t}{t}\right)^{1 / q} \\
& =c_{1}\left(\left.\int_{K_{\varepsilon}^{\alpha}} \frac{\varkappa\left(|x|_{\alpha}\right) f(x)}{\log |x|_{\alpha}}\right|^{q} \frac{\mathrm{d} x}{|x|_{\alpha}^{n}}\right)^{1 / q} \leq c_{1}\left\|f \mid B_{p q}^{n / p, \alpha}\left(\mathbb{R}^{n}\right)\right\| .
\end{aligned}
$$


This time we make use of the extremal functions given by

$$
f_{J}(x):=\sum_{j=1}^{J} J^{-1 / q} \varphi\left(2^{(j-1) \alpha} x\right), \quad x \in \mathbb{R}^{n}, J \in \mathbb{N} .
$$

More details are found in the proof of Theorem 5.1, Step 2, especially (44). Inserting (53) into (52), we obtain a contradiction to our assumption.

The proof of (ii) can be handled as in the proof of Theorem 6.1.

Now, we shall be concerned with an apllication of Theorem 6.2 , in the context of some anisotropic fractals.

Following the recent work of E. Tamási [15], we consider the next definition.

Definition 6.3. Let $\alpha$ be an anisotropy in $\mathbb{R}^{n}$ and let $0<d<n$. A compact set $\Gamma^{\alpha}$ in $\mathbb{R}^{n}$ is called an anisotropic $d$-set if there are a Borel measure $\mu$ in $\mathbb{R}^{n}$ and two positive constants $c_{1}$ and $c_{2}$ such that supp $\mu=\Gamma$ and

$$
c_{1} t^{d} \leq \mu\left(B^{\alpha}(\gamma, t)\right) \leq c_{2} t^{d} \quad \text { for all } 0<t<1 \text { and } \gamma \in \Gamma,
$$

where

$$
B^{\alpha}(\gamma, t):=\left\{x \in \mathbb{R}^{n}: \quad|x-\gamma|_{\alpha}<t\right\}
$$

Let

$$
D_{\alpha}(x):=\operatorname{dist}_{\alpha}\left(x, \Gamma^{\alpha}\right)=\inf _{y \in \Gamma^{\alpha}}|x-y|_{\alpha}
$$

be the anisotropic distance of $x \in \mathbb{R}^{n}$ to $\Gamma^{\alpha}$. Moreover, for $\varepsilon>0$ we define the anisotropic neighbourhood $\Gamma_{\varepsilon}^{\alpha}$ of $\Gamma^{\alpha}$ by

$$
\Gamma_{\varepsilon}^{\alpha}:=\left\{x \in \mathbb{R}^{n}: \quad D_{\alpha}(x)<\varepsilon\right\} .
$$

Proposition 6.4. Let $\alpha$ be an anisotropy in $\mathbb{R}^{n}, 0<d<n$ and let $\Gamma^{\alpha}$ be a compact anisotropic d-set in $\mathbb{R}^{n}$. Moreover, let $1<p<\infty$ and $0<q \leq \infty$. Then

$$
\int_{\Gamma_{\varepsilon}^{\alpha}}\left|\frac{f(x)}{\log D_{\alpha}(x)}\right|^{p} \frac{\mathrm{d} x}{D_{\alpha}^{n-d}(x)} \leq c\left\|f \mid F_{p q}^{n / p, \alpha}\left(\mathbb{R}^{n}\right)\right\|^{p}
$$

for some $c>0$ and all $f \in F_{p q}^{n / p, \alpha}\left(\mathbb{R}^{n}\right)$.

Proof: Consider

$$
\Gamma_{j}:=\left\{x \in \mathbb{R}^{n}: \quad 2^{-\frac{j+1}{n-d}}<D_{\alpha}(x) \leq 2^{-\frac{j}{n-d}}\right\}, \quad j \geq J .
$$


Standard calculations show that $\left|\Gamma_{j}\right| \sim 2^{-j}$. In analogy to (51), we take into account the function

$$
a(x):=\left|\log D_{\alpha}(x)\right|^{-p} D_{\alpha}^{d-n}(x) .
$$

It follows that

$$
a^{*}(t) \sim t^{-1}|\log t|^{-p}, \quad 0<t<\delta<1 .
$$

The rest of the proof runs as the proof of the previous theorem.

\section{References}

[1] C. Bennett and R. Sharpley, Interpolation of Operators, Pure and Appl. Math. 129, Academic Press, New York, 1988.

[2] N. H. Bingham, C. M. Goldie, and J. L. Teugels, Regular variation, Cambridge Univ. Press, 1987.

[3] M. Bricchi and S. D. Moura, Complements on growth envelopes of spaces with generalised smoothness in the sub-critical case, Z. Anal. Anwendungen 22 (2003), no. 2, 383-398.

[4] A. Caetano and W. Farkas, Local growth envelopes of Besov spaces of generalized smoothness, Z. Anal. Anwendungen (to appear).

[5] A. M. Caetano and H.-G. Leopold, Local growth envelopes of Triebel-Lizorkin spaces of generalized smoothness, preprint CM06/I-03, Univ. Aveiro, Portugal, 2005.

[6] A. Caetano and S. D. Moura, Local growth envelopes of spaces of generalized smoothness: the sub-critical case, Math. Nachr. 273 (2004), 43-57.

[7] A. Caetano and S. D. Moura, Local growth envelopes of spaces of generalized smoothness: the critical case, Math. Ineq. Appl. 7 (2004), 573-606.

[8] W. Farkas, Atomic and subatomic decompositions in anisotropic function spaces. Math. Nachr. 209 (2000), 83-113.

[9] W. Farkas, J. Johnsen and W. Sickel, Traces of anisotropic Besov-Lizorkin-Triebel spaces - a complete treatment of the borderline cases, Math. Bohem. 125 (2000), no. 1, 1-37.

[10] D. D. Haroske, Envelopes in function spaces - a first approach, Jenaer Schriften Math. Informatik, Math/Inf/16/01, Jena (2001), 1-72.

[11] D. D. Haroske, Limiting Embeddings, Entropy Numbers and Envelopes in Function Spaces, Habilitationsschrift, Jena, 2002.

$[12]$ D. D. Haroske. Envelopes and sharp embeddings of function spaces. Research Notes in Mathematics. Chapman \& Hall, Boca Raton, 2006 (to appear)

[13] H. J. Schmeisser and H. Triebel, Topics in Fourier Analysis and Function Spaces, John Wiley \& Sons, Chichester, 1987.

[14] W. Sickel and H. Triebel, Hölder inequalities and sharp embeddings in function spaces of $B_{p q}^{s}$ and $F_{p q}^{s}$ type, $Z$. Anal. Anwendungen 14 (1995), no. 1, 105-140.

[15] E. Tamási, Anisotropic Besov spaces and approximation numbers of traces on related fractal sets, Rev. Mat. Complutense (to appear).

[16] H. Triebel, Fourier Analysis and Function Spaces, Teubner, Leipzig, 1977.

[17] H. Triebel, The Structure of Functions, Birkhäuser, Basel, 2001.

[18] H. Triebel, Theory of Function Spaces III, Birkhäuser, Basel, 2006, (to appear).

[19] H. Triebel, Wavelet bases in anisotropic function spaces, Proc. FSDONA-04, Math. Inst. Acad. Czech Republic, 370-387, 2005.

Susana D. Moura 
Department of Mathematics, University of Coimbra, Apartado 3008, 3001-454 Coimbra, PORTUGAL

E-mail address: smpsd@mat.uc.pt

JÚLIO S. NEVES

Department of Mathematics, University of Coimbra, Apartado 3008, 3001-454 Coimbra, PORTUGAL

E-mail address: jsn@mat.uc.pt

Mariusz Piotrowski

Department of Mathematics, University of Coimbra, Apartado 3008, 3001-454 Coimbra, PORTUGAL

E-mail address: marian@mat.uc.pt 